\title{
Effective in vivo and ex vivo gene transfer to intestinal mucosa by VSV-G-pseudotyped lentiviral vectors
}

\author{
Hiroshi Matsumoto', Takahiro Kimura', Kazunori Haga', Noriyuki Kasahara1,2, Peter Anton' and lan McGowan*3
}

\begin{abstract}
Background: Gene transfer to the gastrointestinal (GI) mucosa is a therapeutic strategy which could prove particularly advantageous for treatment of various hereditary and acquired intestinal disorders, including inflammatory bowel disease (IBD), Gl infections, and cancer.

Methods: We evaluated vesicular stomatitis virus glycoprotein envelope (VSV-G)-pseudotyped lentiviral vectors (LV) for efficacy of gene transfer to both murine rectosigmoid colon in vivo and human colon explants ex vivo. LV encoding beta-galactosidase (LV- $\beta$-Gal) or firefly-luciferase (LV-fLuc) reporter genes were administered by intrarectal instillation in mice, or applied topically for ex vivo transduction of human colorectal explant tissues from normal individuals.

Macroscopic and histological evaluations were performed to assess any tissue damage or inflammation. Transduction efficiency and systemic biodistribution were evaluated by real-time quantitative PCR. LV-fLuc expression was evaluated by ex vivo bioluminescence imaging. LV- $\beta$-Gal expression and identity of transduced cell types were examined by histochemical and immunofluorescence staining.

Results: Imaging studies showed positive fLuc signals in murine distal colon; $\beta$-Gal-positive cells were found in both murine and human intestinal tissue. In the murine model, $\beta$-Gal-positive epithelial and lamina propria cells were found to express cytokeratin, CD45, and CD4. LV-transduced $\beta$-Gal-positive cells were also seen in human colorectal explants, consisting mainly of CD45, CD4, and CD11c-positive cells confined to the LP.

Conclusions: We have demonstrated the feasibility of LV-mediated gene transfer into colonic mucosa. We also identified differential patterns of mucosal gene transfer dependent on whether murine or human tissue was used. Within the limitations of the study, the LV did not appear to induce mucosal damage and were not distributed beyond the distal colon.
\end{abstract}

\section{Background}

Gene transfer to the gastrointestinal (GI) mucosa is a therapeutic strategy which could prove particularly advantageous for treatment of various hereditary and acquired intestinal disorders, including inflammatory bowel disease (IBD), GI infections, and cancer [1-5].

Non-viral vectors for delivery of exogenous DNA are limited by low efficiency of transduction in vivo, and do not provide long-term expression [6]. First-generation retroviral vectors can achieve long-term expression through their ability to integrate permanently in the

\footnotetext{
* Correspondence: imcgowan@pitt.edu

${ }^{3}$ Magee-Womens Research Institute, Division of Gastroenterology, Hepatology, and Nutrition, University of Pittsburgh School of Medicine, Pittsburgh, PA, USA Full list of author information is available at the end of the article
}

genome of target cells, but gene transfer to the GI tract by these vectors was also found to be inefficient [7-10]. Conversely, adenoviral vectors can infect a wide range of cells, including intestinal epithelial cells, and show high levels of transgene expression [11,12], but these are non-integrating vectors and so expression is transient [13]. Furthermore, adenoviral vectors induce robust cellular and humoral immune responses in vivo, resulting in elimination of transduced cells and neutralization of the vector upon repeat administration [14]. In contrast, adeno-associated virus (AAV) vectors lack all viral genes and have limited capacity to induce cell mediated immune responses. In addition, AAV may have the potential to 
transduce intestinal crypt progenitor cells resulting in extended transgene expression [15].

$\mathrm{LV}$, such as those derived from human immunodeficiency virus (HIV), are distinct from earlier generation retroviral vectors in their ability to infect quiescent cells through active import of the viral preintegration complex across the intact nuclear membrane, even in post-mitotic cells $[16,17]$. LV pseudotyped with the vesicular stomatitis virus envelope glycoprotein (VSV-G) exhibit an expanded host range that allows entry into most cell types in a wide variety of species ranging from zebrafish to man [18]. Highly efficient gene delivery by VSV-Gpseudotyped LV has been reported in various types of terminally differentiated primary cells in vitro and in vivo, including neurons, hepatocytes, cardiomyocytes, vascular endothelium, alveolar pneumocytes, and keratinocytes [19-29]. With regard to intestinal cells, these vectors have been shown to be capable of transducing human and canine colonic epithelial cell lines via the apical membrane in polarized monolayer cultures in vitro [30]. However, to date there have been no studies evaluating VSVG-pseudotyped LV for gene delivery to primary intestinal cells, especially those subjacent to the mucosal epithelia, particularly in the context of the architecturally complex native tissue.

Therefore, in these studies we tested the ability of VSVG-pseudotyped LV to deliver reporter genes to colonic mucosa via the apical surface in vivo, after intraluminal instillation per rectum in a murine model, and ex vivo in a human intestinal explant system [31].

\section{Methods}

\section{Vector construction}

The pRRLsin-hCMV- $\beta$-Gal vector was constructed by insertion of the $\beta-G$ al reporter gene from plasmid $\mathrm{pSV}-\beta$ Gal (Promega, Madison, WI, USA), and the pRRLsinhCMV-fLuc vector was constructed by insertion of the fLuc gene [32], respectively, into the multiple cloning site (MCS) of pRRLsin-hCMV-MCS-pre, a third-generation, self-inactivating LV construct kindly provided by Dr. Luigi Naldini (University of Milan, Italy) [33] (Figure 1A).

\section{Cell culture, virus production, and in vitro gene transfer}

Human embryonic kidney cell line 293T, and human colon cancer cell lines Caco-2, LoVo, and WiDr, (ATCC, Manassas, VA, USA), were cultured in Dulbecco's modified Eagle's medium (DMEM), Ham's F12K, or RPMI1640 medium, respectively, with $10 \%$ fetal bovine serum and $1 \%$ penicillin-streptomycin. LV virus was produced in $293 \mathrm{~T}$ cells using a third-generation packaging system as previously described [34], using either pRRLsinhCMV- $\beta$-Gal or pRRLsin-hCMV-fLuc, to produce LV- $\beta$ Gal or LV-fLuc, respectively. Virus titers were determined by HIV-1 p24 ELISA (Perkin Elmer, Waltham, MA, USA)

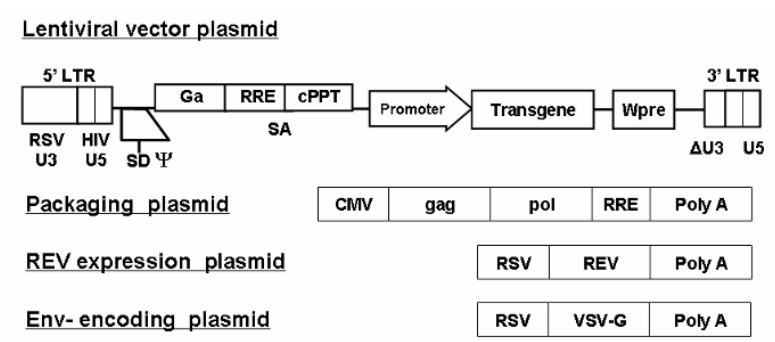

Figure 1 Lentiviral vector (LV) constructs and ex vivo explant culture system. (A) Schematic representation of self-inactivating vectors containing a central polypurine tract (CPPT)/central termination sequence, immediate early cytomegalovirus (CMV) promoter. Vectors were constructed for expression of beta-galactosidase ( $\beta$-Gal) and firefly luciferase (fLuc).

and expressed as p24 equivalent units (ng/ml). Vector transductions were performed on $1 \times 10^{5}$ target cells with $8 \mu \mathrm{g} / \mathrm{mL}$ polybrene (Sigma, St. Louis, MO, USA) at $37^{\circ} \mathrm{C}$. Polybrene is a small, positively charged molecule that binds to cell surfaces and neutralizes surface charge and has been shown to enhance cell transduction by retroviruses [35].

After replacement of the medium and further incubation for $24 \mathrm{hr}$, cells were washed twice in phosphate-buffered saline (PBS), fixed in 2\% formaldehyde/0.2\% glutaraldehyde (Sigma, St. Louis, MO) for $10 \mathrm{~min}$ at room temperature, and stained with $20 \mathrm{mg} / \mathrm{ml} \mathrm{X-Gal} \mathrm{solution}$ (5-bromo-4-chloro-3-indolyl beta-D-galactoside; Sigma, St. Louis, MO, USA) at $37^{\circ} \mathrm{C}$ for $2 \mathrm{hr}$.

\section{In vivo studies}

All in vivo studies were performed according to institutional guidelines under protocols approved by the UCLA Animal Research Committee. Briefly, 6 to 8-week-old female BALB/c mice (Charles River Laboratories Inc., Wilmington, MA, USA) were divided into a non-treated control (NC) group, and two groups that received intrarectal instillation of either LV or vehicle solution (DMEM). Prior to instillation, anesthetized mice were given an intrarectal enema of $50 \%$ ethanol (vol/vol in distilled $\mathrm{H}_{2} \mathrm{O}$ ). Pretreatment with ethanol enemas has been shown to increase intestinal transduction with other vectors [36]. Two hours after the enema, $100 \mu \mathrm{L}$ of vehicle or viral vector solution containing a total of approximately 1000 ng p24 equivalent units was instilled intrarectally. The mice were inverted for $30 \mathrm{sec}$ after administration of intrarectal products to prevent leakage.

\section{Macroscopic assessment and ex vivo bioluminescence imaging}

Health status and body weight was monitored throughout the study. On days 2, 7, and 21 after vector or vehicle instillation, cohorts of mice were sacrificed, and the 
entire colon was removed. Colon length from cecum to anus, weight, and a macroscopic colonic damage score were recorded The macroscopic colonic damage score was based on the degree of tissue adhesion, mucosal ulceration, and intestinal wall thickness (Table 1) [37].

Ex vivo bioluminescence imaging was performed 2 days after rectal instillation of LV-fLuc using the Xenogen IVIS system (Caliper Life Sciences, Alameda, CA, USA). Bioluminescent signal intensity was expressed as photons per second per $\mathrm{cm}^{2}$ per steridian $\left(\mathrm{p} / \mathrm{s} / \mathrm{cm}^{2} / \mathrm{sr}\right)$, and color images were processed with Living Image and IGORPRO analysis software (Wave Metrics, Portland, OR, USA).

\section{Histological evaluation and X-Gal histochemical staining}

For routine histology, colon and other tissues (spleen, liver, lung, kidney) were fixed in $4 \%$ paraformaldehyde overnight, placed in 30\% sucrose/PBS for $2 \mathrm{hr}$, and embedded in OCT compound. Serial $5-\mu \mathrm{m}$ cryosections were stained with hematoxylin/eosin (H\&E) for evaluation of histopathology score. Mucosal inflammation was scored using a semi quantitative technique (Table 2) [38].

For X-Gal histochemistry, tissues were fixed in $2 \%$ glutaraldehyde for $2 \mathrm{hr}$, embedded in OCT compound, and $10-\mu \mathrm{m}$ cryosections were stained in $1 \mathrm{mg} / \mathrm{ml} \mathrm{X-Gal} \mathrm{solu-}$ tion at $37^{\circ} \mathrm{C}$ for $24 \mathrm{sr}$, rehydrated, and counterstained with 0.1\% Nuclear Fast Red (Sigma, St. Louis, MO, USA). The

\section{Table 1: Macroscopic Colonic Damage Score System}

\begin{tabular}{|c|c|}
\hline \multicolumn{2}{|c|}{ Tissue Adhesion } \\
\hline Score & Observation \\
\hline 0 & No adhesion \\
\hline 1 & $\begin{array}{l}\text { Little effort required to separate the colon from the } \\
\text { surrounding tissue }\end{array}$ \\
\hline 2 & $\begin{array}{l}\text { Moderate effort required to separate the colon from } \\
\text { the surrounding tissue }\end{array}$ \\
\hline 3 & Severe adhesions \\
\hline \multicolumn{2}{|c|}{ Degree of ulceration } \\
\hline Score & Observation \\
\hline 0 & Normal appearance of the colon \\
\hline 1 & Focal hyperemia with no ulcer \\
\hline 2 & Presence of an ulcer and inflammation \\
\hline 3 & Two (2) or more ulcers and regions of inflammation \\
\hline \multicolumn{2}{|c|}{ Wall thickness } \\
\hline Score & Observation \\
\hline 0 & Normal thickness \\
\hline 1 & Mild thickening \\
\hline 2 & Moderate thickening \\
\hline 3 & Severe bowel thickening \\
\hline
\end{tabular}

Table 2: Histological Scoring System

\begin{tabular}{|c|c|}
\hline \multicolumn{2}{|c|}{ Infiltration of inflammatory cells } \\
\hline Score & Observation \\
\hline 0 & Rare inflammatory cells in the lamina propria \\
\hline 1 & $\begin{array}{l}\text { Increased numbers of inflammatory cells in the } \\
\text { lamina propria }\end{array}$ \\
\hline 2 & $\begin{array}{l}\text { Confluence of inflammatory cells extending into the } \\
\text { submucosa }\end{array}$ \\
\hline 3 & Transmucosal infiltrates \\
\hline \multicolumn{2}{|c|}{ Tissue Damage } \\
\hline Score & Observation \\
\hline 0 & No mucosal damage \\
\hline 1 & A discrete lymphoepithelial lesion \\
\hline 2 & Surface mucosal erosion \\
\hline 3 & Extensive mucosal damage and ulceration \\
\hline
\end{tabular}

number of positive staining cells was counted in five independent fields in random areas on two non-consecutive slides at $200 \times$ magnification.

\section{Real-time quantitative PCR (qPCR) analysis}

Quantification of vector copy number was performed at each time point by TaqMan qPCR assay to detect the HIV-1 packaging signal sequence, using $300 \mathrm{ng}$ genomic DNA (equivalent to $5 \times 10^{4}$ genomes) isolated from murine colon and other tissues, including stomach, small intestine, liver, kidney, spleen, lung, heart, brain, and bone marrow [39]. A reference curve was prepared by amplifying serial dilutions of $\mathrm{LV}$-encoding plasmid in a background of genomic DNA isolated from untransduced murine colon. Genomic DNA from a PC3 cell line previously confirmed by flow cytometry to be $100 \%$ transduced by LV-GFP vector was used as a positive assay control.

\section{Human colorectal tissue explant culture and ex vivo gene transfer}

All human endoscopic biopsies were collected from healthy HIV-negative volunteers at UCLA Medical Center, Los Angeles, USA. The protocol for the use of human endoscopic biopsies was approved by Institutional Review Board of the David Geffen School of Medicine at UCLA (\#02-05-001-13).

Explant cultures were established as previously described [31]. Briefly, explants were placed on presoaked Gelfoam (Pharmacia and Upjohn Company, Kalamazoo, MI, USA) rafts with the epithelium uppermost. Tissues were transduced by pipetting LV solution and polybrene $(8 \mu \mathrm{g} / \mathrm{mL})$ onto the top of each Gelfoam-supported explant. After $2 \mathrm{hr}$ incubation, tissues were washed three times, placed on fresh Gelfoam rafts, and incubated at 
$37^{\circ} \mathrm{C}$ for a total of $24 \mathrm{hr}$. Explants were then either fixed in glutaraldehyde, embedded in OCT, and 10- $\mu$ m cryosections prepared for X-Gal as above, or were fixed in formaldehyde, embedded in OCT, and $7-\mu \mathrm{m}$ sections prepared for immunofluorescence staining.

\section{Immunofluorescence staining and quantitative morphometry}

The identity of transgene-expressing cells in harvested murine colon tissues or human colorectal explant tissues was examined by immunofluorescence double-staining with antibodies against $\beta$-Gal and cell-specific phenotypic markers. Briefly, serial $7-\mu \mathrm{m}$ cryosections were stained for E. coli $\beta$-Gal (Promega, Madison, WI, USA). Additional staining included antibodies directed against human and murine cytokeratin AE1/AE3 and CD45 (Dako North America Inc., Carpinteria, CA, USA); and CD4, CD8, and CD11c (BD Pharmingen, Franklin Lakes, NJ, USA). $\beta$-Gal staining was then visualized with Alexa Fluor 594. Cytokeratin and CD45 were visualized with Alexa Fluor 488 (Invitrogen Corporation, Carlsbad, CA, USA). The CD4, CD8, and CD11c antibodies were already conjugated with Alexa Fluor 488. The Vector mouse-on-mouse (M.O.M ${ }^{\mathrm{mm}}$ ) Immunodetection Kit (Vector Laboratories, Burlingame, CA, USA) was used to avoid the high background staining caused by antibody binding to endogenous murine immunoglobulin when secondary antibodies were used to amplify primary murine antibodies. Slides were mounted and nuclei counterstained using VECTASHIELD ${ }^{\circ}$ and DAPI (4',6-diamidino-2-phenylindole; Vector Laboratories, Burlingame, CA, USA). In order to quantify LV-mediated $\beta$-Gal transduction efficiency, the total number of cells showing colocalization of $\beta$-Gal-positive and CD45 or CD4 positive signals in 3 randomly selected fields per section at $200 \times$ magnification were identified, and expressed as percentages of the total number of $\beta$-Gal-positive cells or the total number of CD $45+/ C D 4+$ cells.

\section{Statistical analysis}

All values were expressed as means \pm SD. Comparisons between groups were made using the Student $t$-test and the Mann-Whitney $\mathrm{U}$ test, and $p$ values $<0.05$ were considered statistically significant.

\section{Results}

LV-mediated gene transfer to human colorectal cancer cell lines in vitro

LV-mediated transduction efficiency was first assessed in vitro using human colorectal adenocarcinoma cell lines Caco-2, WiDr, and LoVo. In each cell line, a dose-dependent increase in the number of cells showing positive signals upon X-Gal staining was observed with increasing LV concentration (Figure 2A, B). The highest dose tested was $10 \mathrm{ng}$ p24 equivalent units of $\mathrm{LV}$, which corresponds to a biological infectious titer of $10^{6}$ transducing units (TU) on a standardized cell line such as 293T [40]. At this dose, transduction rates per $1 \times 10^{5}$ target cells were $40.6 \%$ for WiDr, $13.7 \%$ for Caco-2, and $13.3 \%$ for LoVo, indicating that although these colon adenocarcinoma cell lines are less permissive for LV compared to 293T embryonic kidney cells, significant gene transfer can be achieved with higher multiplicities of infection. Seppen et al. have previously reported higher rates of transduction in Caco-2 cells but used a transwell system with GFP expressing first and third generation lentiviral vectors [41].

\section{Administration of LV for transduction of murine colorectal mucosa in vivo}

The safety of gene transfer procedures using LV expressing either fLuc or $\beta$-Gal was assessed by both macroscopic and histopathologic criteria in three experimental groups; mice receiving the $\mathrm{LV}$, mice receiving a mock installation (the viral plasmid solution in DMEM), and a control group ( $\mathrm{N}=8$ per condition) Intrarectal administration of $1000 \mathrm{ng}$ p24 equivalent units of VSV-Gpseudotyped LV, corresponding to a $293 \mathrm{~T}$ cell-based standardized biological titer of $10^{8} \mathrm{TU}$, did not affect body weight or induce any gross abnormalities upon routine observation. The ratio of colon weight-to-length was $0.33 \pm 0.04$ in the non-treated control (NC) group, $0.34 \pm$ 0.5 in the mock instillation control group, and $0.27 \pm 0.4$ in the LV instillation group. Thus, there were no signifi-

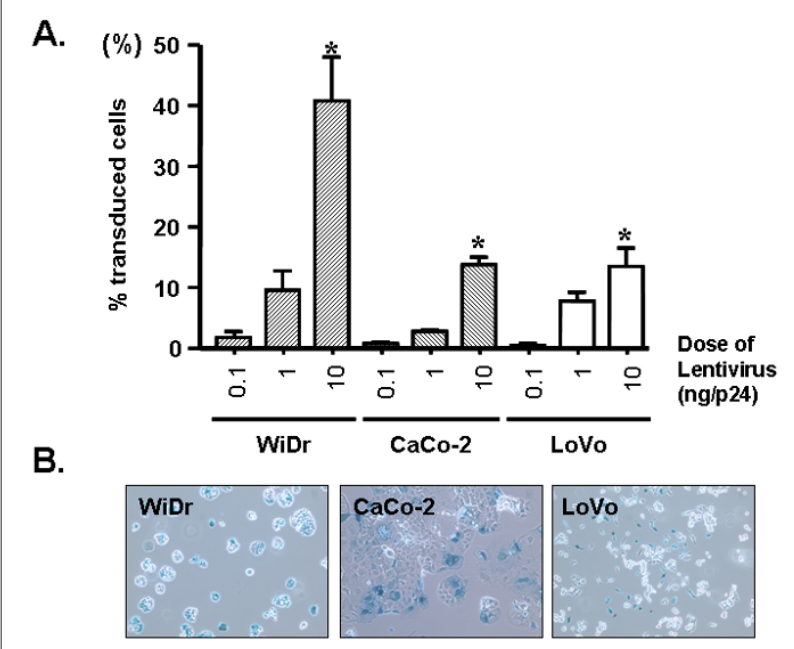

Figure 2 In vitro transduction of vesicular stomatitis virus $G$ protein (VSV-G)-pseudotyped lentivirus (LV) encoding $\beta$-Gal in coIonic cell lines. (A) Percentage of cells transduced by the VSV-Gpseudotyped LV. One hundred cells were counted in three randomly selected, non-adjacent fields in triplicate. (B) Colon adenocarcinoma cell lines showing evidence of $\beta$-Gal transduction following exposure to $10 \mathrm{ng}$ p24 of vector. All values were expressed as means \pm SD. ${ }^{*} \mathrm{p}<$ 0.05 compared with the results of $0.1 \mathrm{ng}$ p24 VSV-G LV transduction. 
cant differences among these three groups in macroscopic damage assessment or histopathological evaluation scores (Additional File 1). More specifically, the use of ethanol enemas did not appear to induce significant mucosal damage. No pathological findings could be observed in kidney, spleen, lung, and liver harvested from control and LV-treated mice at each time point (data not shown).

Ex vivo bioluminescence murine imaging following rectal instillation of LV-fLuc

Strong positive signals were observed in the distal colon adjacent to the rectum in $8 / 8(100 \%)$ of mice exposed to
VSV-G-pseudotyped LV encoding the fLuc reporter gene (Figure 3A). Quantitative measurement of bioluminescent photon emission from LV-transduced distal colon showed a significantly higher level of transduction than that of mock-treated colon $\left(57560 \pm 28960 \mathrm{p} / \mathrm{s} / \mathrm{cm}^{2} / \mathrm{sr}\right.$ for the LV instillation group vs. $6260 \pm 813.3 \mathrm{p} / \mathrm{s} / \mathrm{cm}^{2} / \mathrm{sr}$ for the mock instillation group; $\mathrm{p}<0.001$ ) (Figure $3 \mathrm{~B}$ ). Additional studies are needed to determine whether the physical distribution of the ethanol enemas and/or the LV influenced the location of the bioluminescence signal.
A.

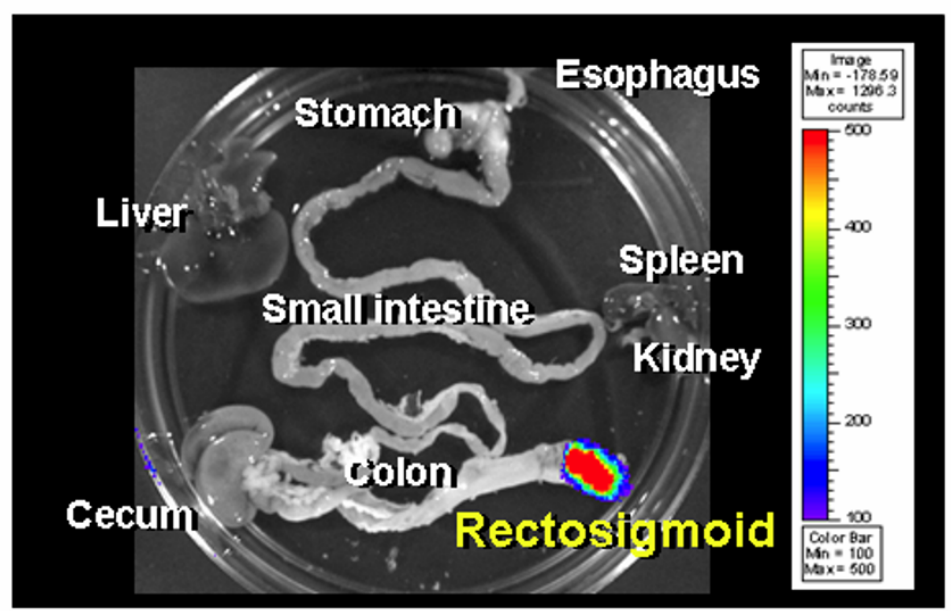

C.

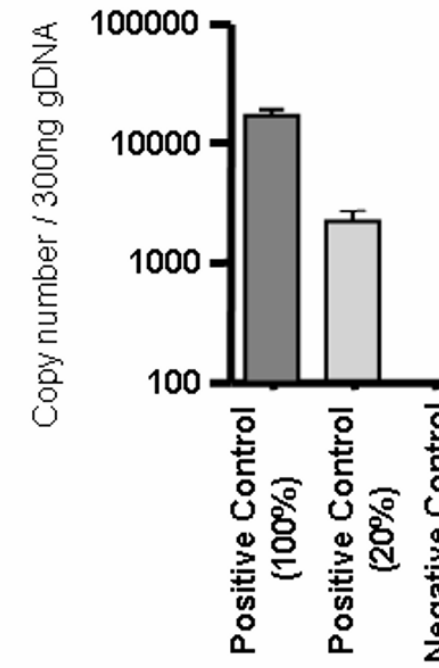

B.

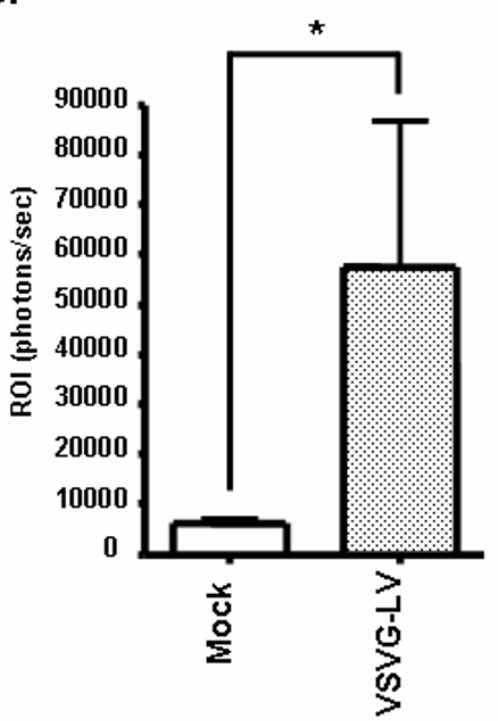


Real-time qPCR analysis of LV transduction in murine colon tissue

Real-time qPCR was employed to quantify the vector copy number of LV integrated into genomic DNA from murine colon tissues after in vivo administration. Using spiked samples to obtain a reference curve, this method was determined to be sensitive enough to detect 50 copies of LV per $5 \times 10^{4}$ cellular genomes. Genomic DNA from both positive control cells (data not shown) and transduced rectosigmoid colon showed amplification of proviral LV sequences. The average copy number per 300 ng genomic DNA from LV-transduced colon was $231.3 \pm$ 183.7 (Figure 3C). As expected, no detectable qPCR signals were observed in genomic DNA from colon tissues of non-treated or mock-treated controls. Importantly, even in LV-treated animals at 2 days post-vector instillation, no detectable qPCR signals were found in any extraintestinal tissues examined, including stomach, small intestine, liver, kidney, spleen, lung, heart, brain, and bone marrow (Figure 3C). As the LV was not treated with DNAse prior to injection, we cannot exclude the possibility that the data in Fig $3 \mathrm{C}$ in part reflect plasmid contamination.

\section{X-Gal histochemistry and double immunofluorescence staining of LV- $\beta$ Gal transduction in murine colon tissues} Histochemistry was performed using optimized X-Gal concentrations and $\mathrm{pH}$ conditions to minimize background staining due to endogenous mammalian $\beta$-galactosidase in the GI tract. As expected, under these conditions, no staining was observed in mouse colon tissues from non-treated and mock-treated control groups, nor in the non-intestinal tissues (kidney, spleen, lung, and liver) from any animals including the LV-treated group.

In contrast, positive-staining cells were observed in colon tissues from animals treated with LV expressing the E. coli $\beta$-Gal reporter gene. The initial number of positive-staining cells in the LV-treated group was $62.3 \pm 18.5$ per microscopic field at $200 \times$ magnification on Day 2 post-vector instillation (Figure 4). However, the number of positive-staining cells was then observed to decrease, to $19.1 \pm 5.3$ per $200 \times$ field on Day 7 , and $15.7 \pm 5.1$ per $200 \times$ field on Day 21 . These positive-staining cells were found predominantly toward the luminal surface of the colon, but were identified in both mucosal epithelium and, importantly, the lamina propria (LP) (Figure 5A).

To further characterize the identity of the transduced cells, immunofluorescence double-staining was performed using an E. coli $\beta$-Gal-specific antibody in combination with various cellular immunophenotypic antibodies. Quantification of double-positive staining in colon tissues harvested on Day 2 post-LV instillation demonstrated that $27 \pm 5 \%$ of the $\beta$-Gal-positive cells were also positive for cytokeratin. Consistent with their

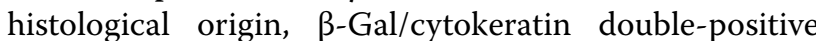
cells were observed solely in the epithelial layers of colon tissues from transduced animals. Cells exhibiting doublepositive staining for both $\beta-\mathrm{Gal}$ and the common leukocyte antigen CD45 were found in both mucosal epithelium and lamina propria (LP) regions. These $\beta$-Gal/CD45 double-positive cells represented $70 \pm 11 \%$ of the total population of $\beta$-Gal-positive cells.

A subset of LV-transduced white blood cells was further identified to consist of $\mathrm{T}$ lymphocytes, dendritic cells, or macrophages, as $27 \pm 2 \%$ of the total population of $\beta$-Gal-positive cells were also positive for CD4. In fact, of the total population of CD4-positive cells, $48 \pm 13 \%$ were $\beta$-Gal-positive, indicating a significant transduction of half of this cell population. These $\beta-G a l / C D 4$ doublepositive cells were observed only within the LP of transduced murine colon. Notably, there were no cells showing co-expression of both $\beta-\mathrm{Gal}$ and CD8 or CD11c (Figure $5 \mathrm{~B}-\mathrm{F})$.

\section{LV- $\beta$ Gal-mediated ex vivo gene transfer to human colorectal explant tissues}

Human colon tissue specimens were incubated with 1000 ng p24 units of LV- $\beta-$ Gal, and examined within $24 \mathrm{hr}$ of explant culture. The number of X-Gal-positive cells after transduction was $12.4 \pm 1.5$ per low power $(\times 200)$ field. In contrast to the murine studies, the transduced cells in human samples were primarily found within the LP (Figure $6 \mathrm{~A}$ ). These data might reflect the availability of both

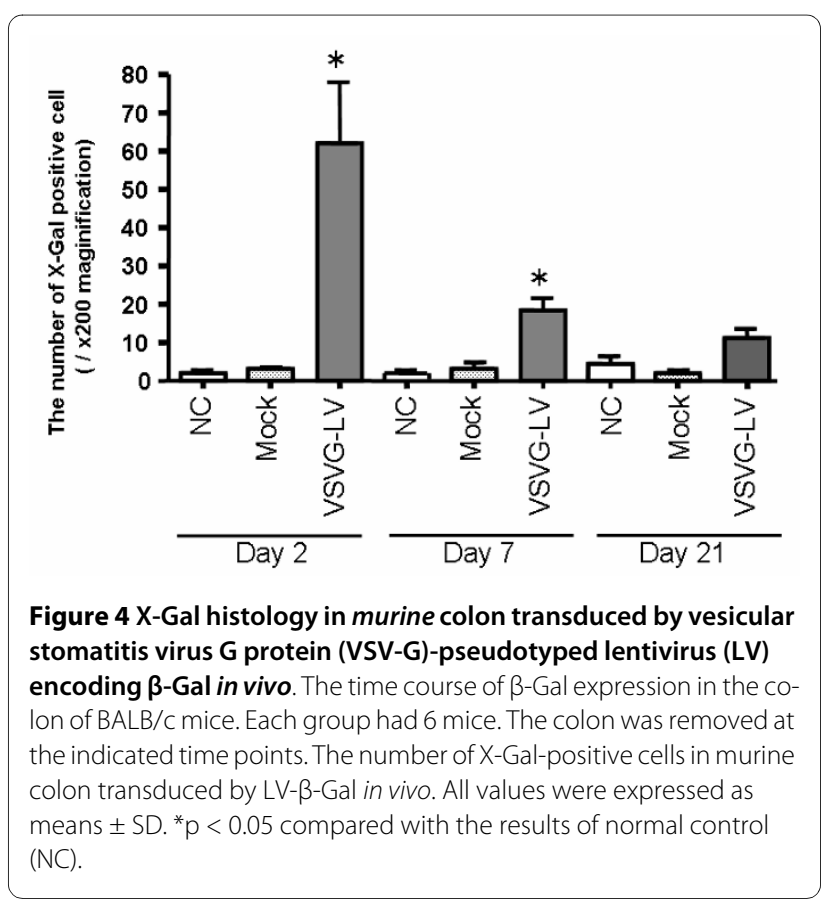



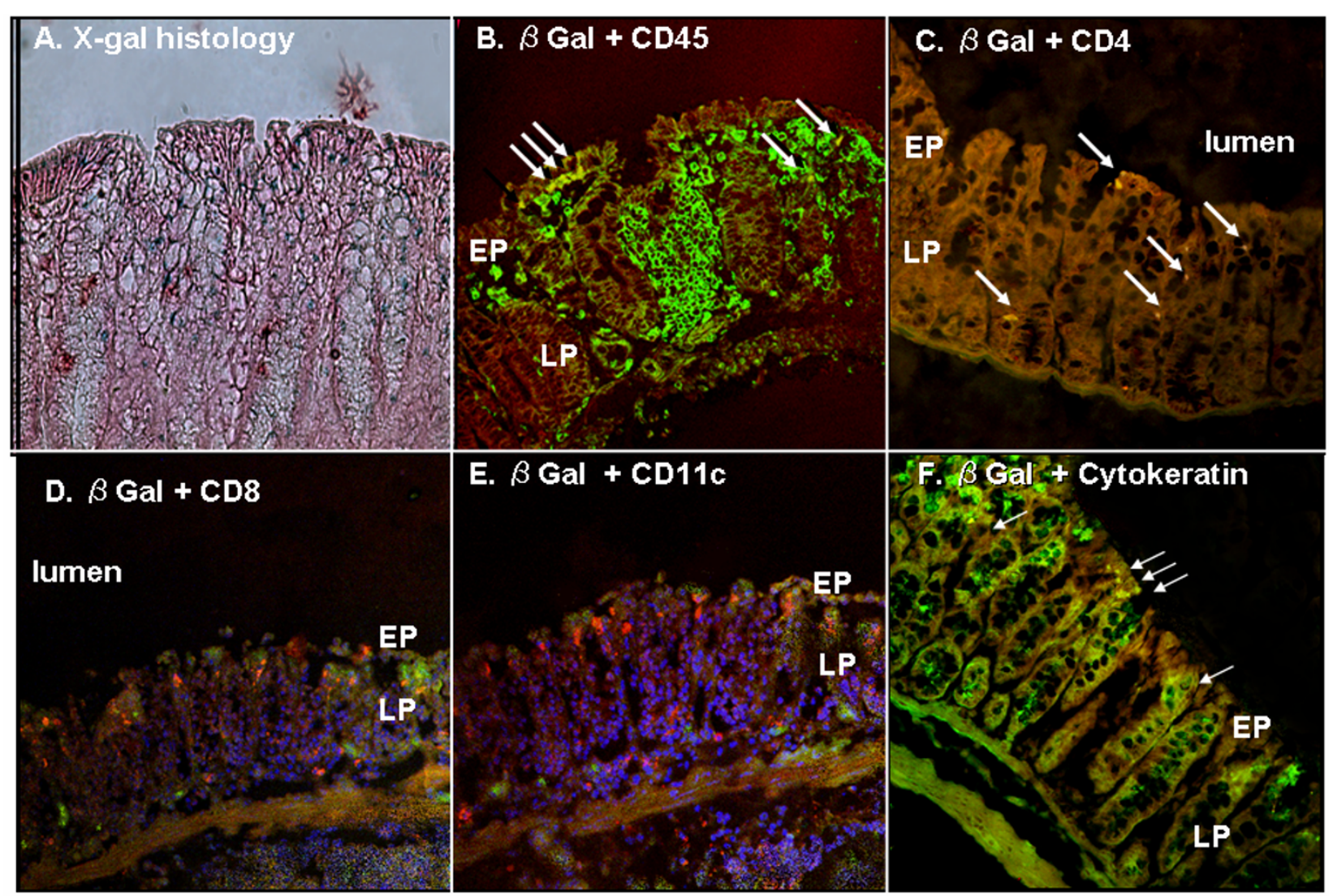

Figure 5 Double immunofluorescence histology analysis in murine colon. (A) Histochemical analysis of transduced murine colon in vivo. The XGal-positive cells were evident mainly on the luminal surface of the distal colon (magnification $\times 400$ ). (B-F) Double immunofluorescence staining of tranduced murine colon examined under confocal microscopy. On Day 2 following rectal administration, $\beta$-Gal-positive cells in murine colon tissue expressed CD45, CD4, and cytokeratin, but not CD8 or CD11c. In these photomicrographs, $\beta$-Gal-positive cells are visualized by red fluorescence, CD phenotypes and cytokeratin are visualized by green fluorescence, and all images display fluorescence overlays: (B) $\beta$-Gal and CD45; (C) $\beta$-Gal and CD4; (D) $\beta$-Gal and CD8; (E) $\beta$-Gal and CD11C; and, (F) $\beta$-Gal and cytokeratin. Double-positive cells indicated by the arrowheads are yellow. (EP; epithelial cell, LP; lamina propria cell)

apical and basal aspects of the explant to LV transduction, and/or the use of polybrene. By double immunofluorescence staining using human cellular immunophenotypic antibodies, $84 \pm 16 \%$ of $\beta$-Gal-positive cells were also found to be positive for CD45. Of these, $41 \pm 12 \%$ of $\beta$-Gal-positive cells were found to be CD4-positive, and $35 \pm 8 \%$ were CD11c-positive. Notably, no $\boldsymbol{\beta}$-Gal-positive cells expressed CD8 or cytokeratin (Figure 6B-F). Non-specific background staining was not observed using this technique (Additional File 2). This identifies transduced cells as mucosal lamina propria CD4+ lymphocytes, macrophages, dendritic cells, and other leukocytes. Consistent with the results obtained after in vivo LV instillation in mice, $54 \pm 15 \%$ of the total population of CD4-positive cells in ex vivo LV-transduced human explant tissues were also positive for $\boldsymbol{\beta}$-Gal. We did not perform immunohistochemical examination of mesenteric lymph node tissue and so we do not know whether LV transduced cells could be identified at this site.

\section{Discussion}

Using a combination of molecular, imaging, and histological techniques, we have demonstrated for the first time that VSV-G-pseudotyped LV can mediate detectable gene transfer to architecturally intact human and murine intestinal mucosa after topical application. The overall transduction level was observed to decrease over time, with a 3-fold reduction between Day 2 and Day 7, but was then relatively consistent between Day 7 and Day 21 . This may reflect both initial transgene expression from unintegrated episomal reverse-transcribed circular forms of LV provirus, which is transient in duration, as well as reduced numbers of transduced non-adherent cells (such 


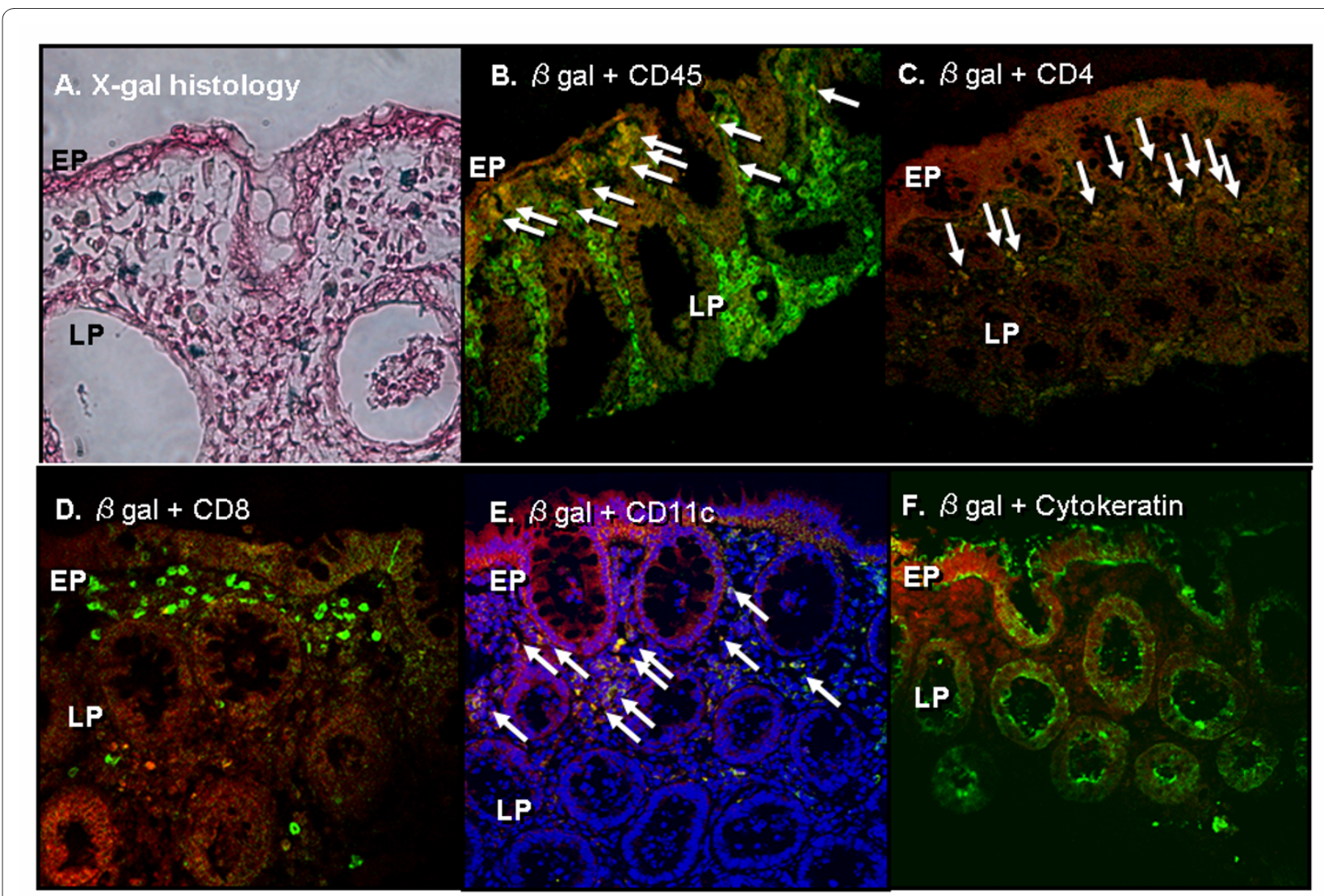

Figure 6 X-Gal histology and double immunofluorescence histology analysis in human colonic explants transduced by VSV-G-pseudotyped lentivirus (LV) encoding $\beta$-Gal. (A) Histological appearance of transduced human explants after X-Gal staining. X-Gal-positive cells, here stained blue, were found mainly in the LP. (B-F) Double immunofluorescence confocal microscopy of human explants transduced with VSV-G. $\beta$-Galpositive cells expressed CD4, CD45, and CD11C, but not CD8, or cytokeratin. In these photomicrographs, $\beta$-Gal is visualized by red fluorescence, each cellular phenotype marker is visualized by green fluorescence, and nuclei are visualized by blue DAPI staining, respectively, and all images display fluorescence overlays: (B) $\beta$-Gal and CD45; (C) $\beta$-Gal and CD4; (D) $\beta$-Gal and CD8; (E) $\beta$-Gal and CD11 c; and, (F) $\beta$-Gal and cytokeratin. Double-positive cells, indicated by the arrowheads, are yellow (EP; epithelial cell, LP; lamina propria cell).

as lymphocytes) as they migrate or circulate out of colon tissue in vivo, or reflect cells lost through apoptosis or karyolysis in the ex vivo explant model.

An additional finding is the demonstration that in human intestinal tissue, the transduced cells are predominantly in the lamina propria, an observation differing from previous beliefs that it is predominantly epithelial lineage cells that are transduced. Co-localization of phenotypic cell surface markers and transgene expression showed notable differences in the types of cells transduced between experiments involving LV-mediated transduction of murine vs. human colon tissue. Significant transduction of cytokeratin-positive cells was observed after in vivo transduction of murine colon, but not after ex vivo transduction of human explants colon tissue. Conversely, after ex vivo transduction of human explant colon tissue, but not after in vivo transduction of murine colon, we observed significant transduction of cells positive for $\mathrm{CD} 11 \mathrm{c}$, a cellular marker of mac- rophages and dendritic cells, but which is also weakly expressed on B cells, NK cells, and T cell subsets.

A number of parameters may have contributed to these differences. For example, to avoid degradation of the tissue architecture, LV-transduced human colon tissues had to be analyzed after no more than 24 hours of explant culture, and the time course for accumulating a detectable level transgene product may require a longer time interval for epithelial cells. Of course, LV transduction after rectal instillation in vivo also necessitates penetration from the intestinal lumen, and the mucosal epithelium represents the only tissue surface directly in contact with the virus solution. In contrast, virus applied ex vivo to the surface of endoscopically acquired human tissue explant samples has access to sub-epithelial cell layers that would not normally be available to virus administered intraluminally to intact intestine in vivo. Another difference between the in vivo and ex vivo experiments was the use of ethanol in the former, and polybrene in the latter, to 
facilitate lentiviral transduction. We did not explore the extent to which these different experimental parameters might influence the results of our experiments.

We did observe a difference between the present study and previous studies using explant tissues, in terms of the specific localization of transduced cells within the LP. In the present study, LV-transduced cells in human explant samples were found primarily in the subepithelial region of the LP whereas in other colorectal explant studies using adenoviral vectors [42] transduction was commonly observed in the basolateral region. However, it is difficult to compare our data with other published studies as the cell tropism may differ between vectors. In addition, differences in the histological distribution of vectorspecific cellular receptors, such as the coxsackievirus/ adenovirus receptor (CAR) required for efficient binding of adenovirus to target cells may influence experimental results.

There were also similarities in cell transduction results from the murine rectal in vivo transduction and human explant ex vivo transduction models. In both cases, VSVG-pseudotyped LV exhibited the ability to transduce CD4-positive cell population. Notably, LV pseudotyped with the VSV-G envelope, which only requires binding to phospholipid constituents of most mammalian cells [43], achieved much more efficient infection of $\mathrm{T}$ cells than is possible with identical vectors pseudotyped with amphotropic retrovirus envelope [44]. However, CD4 is not only expressed by helper $\mathrm{T}$ lymphocytes and $\mathrm{T}$ regulatory cells, but also by macrophages and dendritic cells at lower levels. All of these cell types generally reside in the LP, which is where the predominant staining was observed in humans. It is not clear why the LV was unable to transduce CD8 positive cells and this finding warrants additional in vitro studies with purified $\mathrm{T}$ cell populations.

As the vectors used are replication-defective and can only mediate a single cycle of infection, this finding suggests that LV may be efficiently transported intact across the mucosal epithelium as has been suggested as a natural route of HIV infection. It has also been reported that dendritic cells may form projections into the intestinal lumen to sample incoming antigens, which may permit HIV infection as well as LV transduction of this cell population.

\section{Conclusions}

In summary, these studies have demonstrated the feasibility of using VSV-G-pseudotyped LV to safely achieve appreciable levels of localized gene transfer into architecturally intact primary murine and human intestinal tissues.

\section{Additional material}

Additional file 1 The effect of vesicular stomatitis virus G protein (VSV-G)pseudotyped lentivirus (LV) rectal gene transduction on healthy murine cells. BALB/c mice were divided into three groups; a normal healthy control (NC) group and two groups that received either placebo or VSV-G LV following a preliminary ethanol enema (EtOH). Mice received $1000 \mathrm{ng}$ p24 VSV-G LV by rectal administration under anesthesia. (A) VSV-G LV did not affect weight loss in healthy mice. Results are shown as a percentage of original weight for each group. (B) There was no significant difference in the ratio of colon length to weight between groups. Further, there was no significant difference in (C) macroscopic damage score or (D) histomorphologic score among all groups.

Additional file $\mathbf{2}$ The negative control pictures of immunofluorence staining using AF488 secondary antibody. Non-specific green staining is not observed in either (A) AE1/AE3 or (B) CD45 examination of murine intestinal tissue or (C) AE1/AE3 (D) CD45 examination of exvivo human explant tissue

\section{Competing interests}

The authors declare that they have no competing interests.

\section{Authors' contributions}

HM conducted the majority of the experiments described in this paper. TK carried out the molecular genetic studies, made the LV, and performed the statistical analysis of the experimental data. $\mathrm{KH}$ helped to analyze the in vivo animal data. NK provided technical assistance for the LV experiments, PA collected the endoscopic biopsies, and IM supervised the research, and edited the final manuscript. All authors read and approved the final manuscript.

\section{Acknowledgements}

The authors would like to thank the staff of the Center for HIV Prevention Research (CPR), the UCLA Vector Core \& Shared Resource, and the Blinder Research Foundation for Crohn's Disease. HM was supported by a fellowship from the Blinder Research Foundation for Crohn's Disease, Los Angeles, CA. Additional support was provided by a Pilot Award from the UCLA AIDS Institute (IM, NK), funding from the CURE Digestive Diseases Research Center (P30 DK41301) and Jonsson Comprehensive Cancer Center (P30 CA82103) to the Vector Core \& Shared Resource (NK), and the CFAR Mucosal Immunology Core (Al28697).

\section{Author Details}

'Department of Medicine, Division of Digestive Diseases, University of California Los Angeles (UCLA) David Geffen School of Medicine, Los Angeles, CA, USA, 2Department of Molecular \& Medical Pharmacology, University of California Los Angeles (UCLA) David Geffen School of Medicine, Los Angeles, CA, USA and ${ }^{3}$ Magee-Womens Research Institute, Division of Gastroenterology, Hepatology, and Nutrition, University of Pittsburgh School of Medicine, Pittsburgh, PA, USA

Received: 30 June 2009 Accepted: 11 May 2010 Published: 11 May 2010

\section{References}

1. Prieto J, Herraiz M, Sangro B, Qian C, Mazzolini G, Melero I, et al:: The promise of gene therapy in gastrointestinal and liver diseases. Gut 2003, 52(Suppl 2):ii49-ii54

2. Raper SE, Wilson JM: Making space for intestinal gene therapy. Gastroenterology 1997, 112:1753-1756.

3. Forbes SJ, Hodgson $\mathrm{HJ}$ : Review article: gene therapy in gastroenterology and hepatology. Aliment Pharmacol Ther 1997 11:823-836.

4. van MC, Te Velde AA, venter SJ van De, Rodriguez Pena MS: Gene therapy in the treatment of intestinal inflammation. Int J Colorectal Dis 2004, 19:79-86.

5. Wirtz S, Neurath MF: Gene transfer approaches for the treatment of inflammatory bowel disease. Gene Ther 2003, 10:854-860.

6. Schmid RM, Weidenbach H, Draenert GF, Lerch MM, Liptay S, Schorr J, et al:: Liposome mediated in vivo gene transfer into different tissues of the gastrointestinal tract. Z Gastroenterol 1994, 32:665-670. 
7. Lau C, Soriano HE, Ledley FD, Finegold MJ, Wolfe JH, Birkenmeier EH, et al:: Retroviral gene transfer into the intestinal epithelium. Hum Gene Ther 1995, 6:1145-1151.

8. Noel RA, Shukla P, Henning SJ: Optimization of gene transfer into intestinal epithelial cells using a retroviral vector. J Pediatr Gastroenterol Nutr 1994, 19:43-49.

9. Lozier JN, Yankaskas JR, Ramsey WJ, Chen L, Berschneider H, Morgan RA: Gut epithelial cells as targets for gene therapy of hemophilia. Hum Gene Ther 1997, 8:1481-1490

10. Laine F, Blouin V, Ferry N: Evaluation of recombinant retrovirus and adenovirus for gene transfer to normal and pathologic intestinal tissue. Gastroenterol Clin Biol 1999, 23:221-228.

11. Kesisoglou F, Schmiedlin-Ren P, Fleisher D, Roessler B, Zimmermann EM: Restituting intestinal epithelial cells exhibit increased transducibility by adenoviral vectors. J Gene Med 2006, 8:1379-1392.

12. Brown GR, Thiele DL, Silva M, Beutler B: Adenoviral vectors given intravenously to immunocompromised mice yield stable transduction of the colonic epithelium. Gastroenterology 1997, 112:1586-1594.

13. Russell WC: Update on adenovirus and its vectors. J Gen Viro/ 2000 , 81:2573-2604

14. Bessis N, GarciaCozar FJ, Boissier MC: Immune responses to gene therapy vectors: influence on vector function and effector mechanisms. Gene Ther 2004, 11(Suppl 1):S10-S17.

15. Polyak S, Mah C, Porvasnik S, Herlihy JD, Campbell-Thompson M, Byrne BJ, et al: Gene delivery to intestinal epithelial cells in vitro and in vivo with recombinant adeno-associated virus types 1, 2 and 5. Dig Dis Sci 2008, 53:1261-1270

16. Bukrinsky MI, Haffar OK: HIV-1 nuclear import: in search of a leader. Front Biosci 1999, 4:D772-D781.

17. Zennou V, Petit C, Guetard D, Nerhbass U, Montagnier L, Charneau P: HIV1 genome nuclear import is mediated by a central DNA flap. Cell 2000, 101:173-185.

18. Schubert M, Joshi B, Blondel D, Harmison GG: Insertion of the human immunodeficiency virus CD4 receptor into the envelope of vesicular stomatitis virus particles. J Virol 1992, 66:1579-1589.

19. Blomer U, Naldini L, Verma IM, Trono D, Gage FH: Applications of gene therapy to the CNS. Hum Mol Genet 1996, 5(Spec No):1397-1404

20. Sakoda T, Kasahara N, Hamamori Y, Kedes L: A high-titer lentiviral production system mediates efficient transduction of differentiated cells including beating cardiac myocytes. J Mol Cell Cardiol 1999, 31:2037-2047

21. Shichinohe T, Bochner BH, Mizutani K, Nishida M, Hegerich-Gilliam S, Naldini $L$, et al: Development of lentiviral vectors for antiangiogenic gene delivery. Cancer Gene Ther 2001, 8:879-889.

22. Borok Z, Harboe-Schmidt JE, Brody SL, You Y, Zhou B, Li X, et al.: Vesicular stomatitis virus $G$-pseudotyped lentivirus vectors mediate efficient apical transduction of polarized quiescent primary alveolar epithelial cells. J Virol 2001, 75:1 1747-11754

23. Li W, Nadelman C, Gratch NS, Li W, Chen M, Kasahara N, et al:: An important role for protein kinase C-delta in human keratinocyte migration on dermal collagen. Exp Cell Res 2002, 273:219-228.

24. Chen M, Li W, Fan J, Kasahara N, Woodley D: An efficient gene transduction system for studying gene function in primary human dermal fibroblasts and epidermal keratinocytes. Clin Exp Dermatol 2003, 28:193-199.

25. Naldini L, Blomer U, Gage FH, Trono D, Verma IM: Efficient transfer, integration, and sustained long-term expression of the transgene in adult rat brains injected with a lentiviral vector. Proc Nat I Acad Sci USA 1996, 93:11382-11388

26. Kafri T, Blomer U, Peterson DA, Gage FH, Verma IM: Sustained expression of genes delivered directly into liver and muscle by lentiviral vectors. Nat Genet 1997, 17:314-317.

27. Miyoshi H, Smith KA, Mosier DE, Verma IM, Torbett BE: Transduction of human $\mathrm{CD} 34+$ cells that mediate long-term engraftment of NOD/SCID mice by HIV vectors. Science 1999, 283:682-686.

28. Johnson LG, Olsen JC, Naldini L, Boucher RC: Pseudotyped human lentiviral vector-mediated gene transfer to airway epithelia in vivo. Gene Ther 2000, 7:568-574.

29. Kremer KL, Dunning KR, Parsons DW, Anson DS: Gene delivery to airway epithelial cells in vivo: a direct comparison of apical and basolateral transduction strategies using pseudotyped lentivirus vectors. J Gene Med 2007, 9:362-368.
30. Seppen J, Barry SC, Klinkspoor JH, Katen LJ, Lee SP, Garcia JV, et al:: Apical gene transfer into quiescent human and canine polarized intestinal epithelial cells by lentivirus vectors. J Virol 2000, 74:7642-7645

31. Fletcher PS, Elliott J, Grivel JC, Margolis L, Anton P, McGowan I, et al.: Ex vivo culture of human colorectal tissue for the evaluation of candidate microbicides. AIDS 2006, 20:1237-1245.

32. Koya RC, Kimura T, Ribas A, Rozengurt N, Lawson GW, Faure-Kumar E, et al: Lentiviral vector-mediated autonomous differentiation of mouse bone marrow cells into immunologically potent dendritic cell vaccines. Mol Ther 2007, 15:971-980.

33. Zufferey R, Dull T, Mandel RJ, Bukovsky A, Quiroz D, Naldini L, et al:: Selfinactivating lentivirus vector for safe and efficient in vivo gene delivery. J Virol 1998, 72:9873-9880.

34. Dull T, Zufferey R, Kelly M, Mandel RJ, Nguyen M, Trono D, et al:: A thirdgeneration lentivirus vector with a conditional packaging system. $J$ Virol 1998, 72:8463-8471.

35. Landazuri N, Le Doux JM: Complexation of retroviruses with charged polymers enhances gene transfer by increasing the rate that viruses are delivered to cells. J Gene Med 2004, 6:1304-1319.

36. Vallance BA, Gunawan Ml, Hewlett B, Bercik P, Van KC, Galeazzi F, et al. TGF-beta1 gene transfer to the mouse colon leads to intestinal fibrosis. Am J Physiol Gastrointest Liver Physiol 2005, 289:G1 16-G128.

37. Wallace JL, Keenan CM, Gale D, Shoupe TS: Exacerbation of experimental colitis by nonsteroidal anti-inflammatory drugs is not related to elevated leukotriene B4 synthesis. Gastroenterology 1992, 102:18-27.

38. Cooper HS, Murthy SN, Shah RS, Sedergran DJ: Clinicopathologic study of dextran sulfate sodium experimental murine colitis. Lab Invest 1993, 69:238-249

39. Sastry L, Johnson T, Hobson MJ, Smucker B, Cornetta K: Titering lentiviral vectors: comparison of DNA, RNA and marker expression methods. Gene Ther 2002, 9:1155-1162.

40. Stripecke R, Koya RC, Ta HQ, Kasahara N, Levine AM: The use of lentiviral vectors in gene therapy of leukemia: combinatorial gene delivery of immunomodulators into leukemia cells by state-of-the-art vectors. Blood Cells Mol Dis 2003, 31:28-37.

41. Seppen J, Barry SC, Klinkspoor JH, Katen LJ, Lee SP, Garcia JV, et al.: Apical gene transfer into quiescent human and canine polarized intestinal epithelial cells by lentivirus vectors. J Virol 2000, 74:7642-7645.

42. Schmiedlin-Ren P, Kesisoglou F, Mapili JA, Sabek SE, Barnett JL, Chey WD, et al: Increased transduction of human intestinal epithelial cells by adenoviral vectors in inflammatory bowel disease. Inflamm Bowel Dis 2005, 11:464-472.

43. Yee JK, Friedmann T, Burns JC: Generation of high-titer pseudotyped retroviral vectors with very broad host range. Methods Cell Biol 1994, 43(Pt A):99-112

44. Sharma S, Cantwell M, Kipps TJ, Friedmann T: Efficient infection of a human T-cell line and of human primary peripheral blood leukocytes with a pseudotyped retrovirus vector. Proc Natl Acad Sci USA 1996 93:11842-11847.

Pre-publication history

The pre-publication history for this paper can be accessed here: http://www.biomedcentral.com/1471-230X/10/44/prepub

doi: 10.1186/1471-230X-10-44

Cite this article as: Matsumoto et al., Effective in vivo and ex vivo gene transfer to intestinal mucosa by VSV-G-pseudotyped lentiviral vectors BMC Gastroenterology 2010, 10:44 